\title{
Predictive Factors for Good Outcome and Mortality After Stent-Retriever Thrombectomy in Patients With Acute Anterior Circulation Stroke
}

\author{
Woong Yoon, ${ }^{a}$ Seul Kee Kim, ${ }^{a}$ Man Seok Park, ${ }^{b}$ Byung Hyun Baek, ${ }^{a}$ Yun Young Lee ${ }^{\mathrm{a}}$ \\ Departments of ${ }^{\text {Radiology, }}{ }^{b}$ Neurology, Chonnam National University Medical School, Chonnam National University Hospital, Gwangju, Korea
}

Background and Purpose Predictive factors associated with stent-retriever thrombectomy for patients with acute anterior circulation stroke remain to be elucidated. This study aimed to investigate clinical and procedural factors predictive of good outcome and mortality after stent-retriever thrombectomy in a large cohort of patients with acute anterior circulation stroke.

Methods We analyzed clinical and procedural data in 335 patients with acute anterior circulation stroke treated with stent-retriever thrombectomy. A good outcome was defined as a modified Rankin Scale score of 0 to 2 at 3 months. The associations between clinical, imaging, and procedural factors and good outcome and mortality, respectively, were evaluated using logistic regression analysis.

Results Using multivariate analysis, age (odds ratio [OR], 0.965; 95\% confidence interval [Cl], 0.9440.986; $P=0.001$ ), successful revascularization (OR, 4.658; 95\% Cl, 2.240-9.689; $P<0.001$ ), parenchymal hemorrhage (OR, 0.150; 95\% Cl, 0.049-0.460; $P=0.001)$, and baseline NIHSS score (OR, 0.908; $95 \% \mathrm{Cl}, 0.855-0.965 ; P=0.002$ ) were independent predictors of good outcome. Independent predictors of mortality were age $(0 \mathrm{R}, 1.043 ; 95 \% \mathrm{Cl}, 1.002-1.086 ; P=0.041)$, successful revascularization (OR, 0.171; 95\% Cl, 0.079-0.370; $P<0.001$ ), parenchymal hemorrhage (OR, 2.961; $95 \% \mathrm{Cl}, 1.059$ 8.276; $P=0.038$ ), and a history of previous stroke/TIA (OR, 3.124; $95 \% \mathrm{Cl}, 1.340-7.281 ; P=0.008)$.

Conclusions Age, revascularization status, and parenchymal hemorrhage are independent predictors of both good outcome and mortality after stent retriever thrombectomy for acute anterior circulation stroke. In addition, NIHSS score on admission is independently associated with good outcome, whereas a history of previous stroke is independently associated with mortality.

Keywords Brain infarction; Thrombectomy; Prognosis; Mortality; Outcome
Correspondence: Woong Yoon Department of Radiology, Chonnam National University Hospital, 42 Jebong-ro, Dong-gu, Gwangju 61469, Korea

Tel: +82-62-220-5746

Fax: +82-62-226-4380

E-mail: radyoon@jnu.ac.kr

Received: June 30, 3016

Revised: August 22, 2016

Accepted: December 29, 2016

This study was supported by a grant (CRI15901-22.) from Chonnam National University Hospital Biomedical Research Institute.

The authors have no financial conflicts of interest.

\section{Introduction}

Based on the results of 5 randomized clinical trials (RCTs), mechanical thrombectomy using the stent-retriever has been approved as standard treatment for acute anterior circulation stroke due to occlusions of the internal carotid artery (ICA) or the M1 segment of the middle cerebral artery (MCA). ${ }^{1}$ Recent studies regarding meta-analysis of the 5 RCTs showed that stent-retriever thrombectomy is associated with considerable improvement of functional independence compared with standard medical care. ${ }^{2,3}$

After its acceptance as an effective treatment option, the next steps will be to further establish patient selection criteria and to generalize stent-retriever thrombectomy to a broader class of stroke patients with acute large vessel occlusions. To further broaden treatment indications for stent-retriever thrombectomy, understanding the prognostic factors related to clinical outcomes could be essential. Although there exist a few studies that have investigated prognostic factors that predict outcomes after 
stent retriever thrombectomy in patients with acute anterior circulation stroke, these studies are limited by small sample size and the inclusion of patients with posterior circulation stroke. ${ }^{4-8}$ Thus, this study aimed to investigate clinical, imaging, and procedural factors predictive of good outcome and mortality after stent-retriever thrombectomy in a large cohort of patients with acute anterior circulation stroke.

\section{Methods}

\section{Patients}

From December 2010 to November 2015, a total of 356 consecutive patients presenting with acute ischemic stroke due to ICA or MCA occlusions were treated with stent-retriever thrombectomy at a comprehensive regional stroke center. Patients underwent a non-enhanced CT scan and multimodal MRI before endovascular treatment. MRI examinations were performed using a 1.5-T unit (Signa HDxt; GE Medical Systems, Milwaukee, USA). MRI sequences included DWI, gradient echo imaging, FLAIR sequence, 3-dimensional TOF MRA, and perfusion imaging. DWI sequences were obtained in the axial plane using a single-shot, spin-echo echo-planar technique with following parameters: TR of 9,000 ms, TE of $80 \mathrm{~ms}$, slice thickness of $4 \mathrm{~mm}$, interslice gap of $0 \mathrm{~mm}$, FOV of $260 \times 260 \mathrm{~mm}$, and $b$ values of 0 and $1,000 \mathrm{sec} / \mathrm{mm}^{2}$.

Of 356 patients, those who had a prestroke modified Rankin Scale (mRS) score $\geq 3$ ( $n=11)$ or concomitant posterior circulation infarctions $(n=8)$ or who were lost to follow-up $(n=2)$ were excluded, leaving 335 patients in this study. We prospectively collected the following clinical, imaging, and procedural data of these 335 patients: demographic features, cerebrovascular risk factors, NIHSS scores on admission, use of IV thrombolysis, time to endovascular treatment, procedure time, time to reperfusion, revascularization status, underlying intracranial atherosclerotic stenosis, tandem occlusion at the proximal cervical portion of the ICA, intracranial hemorrhage on post-therapeutic CT scans or gradient echo MRI, Alberta Stroke Prognosis Early CT score (ASPECTS) applied to DWI (DWI-ASPECTS), and clinical outcomes at 3 months. The institutional ethics committee approved this retrospective analysis and waived informed consent based on the study design.

\section{Endovascular treatment}

Upon admission, a stroke neurologist performed a neurological assessment based on the NIHSS. The inclusion criteria for stentretriever embolectomy were as follows: (1) the procedure started within 6 hours of symptom onset, (2) baseline NIHSS score $\geq 4$, (3) occlusion of ICA or MCA, (4) no intracranial hemorrhage detected on the cranial $\mathrm{CT}$ or MRI, and (5) infarct volume less than one-third of the MCA territory on DWI (determined as DWI-ASPECTS $>3$ ) or non-enhanced CT (determined by Alteplase Thrombolysis for Acute Noninterventional Therapy in Ischemic Stroke [ATLANTIS] criteria). ${ }^{9}$

For each patient, written informed consent for endovascular therapy was obtained from a family member. Endovascular therapy was performed under conscious sedation. In cases of agitation, an intravenous bolus of midazolam was given and repeated if necessary. Stent-based thrombectomy with a Solitaire stent (Covidien, Irvine, USA) or Trevo stent (Stryker, Kalamazoo, USA) was performed as the first-line endovascular treatment. When stent-based thrombectomy was unsuccessful, additional mechanical approaches were performed, including manual aspiration thrombectomy with a Penumbra reperfusion catheter (Penumbra, Alameda, USA) or Navien catheter (Covidien, Irvine, USA). The details of the techniques used for mechanical thrombectomy were previously described. ${ }^{10,11}$ Intracranial angioplasty with or without stenting was performed when severe $(>70 \%)$ underlying intracranial atherosclerotic stenosis was observed. When the patient had a tandem occlusion at the proximal cervical portion of the ICA, carotid angioplasty and stenting were performed prior to stent-retriever thrombectomy. Revascularization status was assessed on the final angiogram and was classified according to the modified $\mathrm{TICl}$ scale, and successful revascularization was defined as a modified $\mathrm{TICl}$ grade of $2 \mathrm{~b}$ or $3 .^{12}$ All patients underwent non-enhanced CT scans immediately and 24 hours after endovascular treatment and gradient echo MRI 24-36 hours after treatment. Intracerebral hemorrhages were assessed on posttreatment $\mathrm{CT}$ and gradient echo MR images and classified as hemorrhagic infarction $(\mathrm{HI})$ or parenchymal hemorrhage (PH) based on the European Cooperative Acute Stroke Study (ECASS) criteria. ${ }^{13}$ Symptomatic intracranial hemorrhage was defined as any intracranial hemorrhage that caused neurological deterioration (increase of $\geq 4$ points in NIHSS score or deterioration of 1 point in the level of consciousness on NIHSS). Clinical outcome was assessed by a stroke neurologist using the modified Rankin Scale (mRS) during an outpatient visit 3 months after treatment. If patients were unable to attend the outpatient clinic, outcomes were obtained via a telephone interview. A good clinical outcome was defined as an $\mathrm{mRS}$ score $\leq 2$.

\section{Statistical analysis}

Continuous variables are presented as medians and interquartile ranges (IQR). Discrete variables are presented as counts ( $n$ ) and percentages (\%). First, the relationship between each clinical and procedural characteristic and 3-month outcome was determined. The $\chi^{2}$ test or Fisher exact test was used for comparing categorical variables, and the Mann-Whitney $U$ test was used 
for comparing continuous variables. Second, independent associations between good outcome (mRS 0-2) and clinical and procedural factors were determined with a multivariate logistic regression analysis. The variables tested in the multivariate logistic regression models were those with $P<0.05$ in the univariate analysis. Third, a multivariate logistic regression analysis was performed to identify independent predictors for mortality at 3 months. Variables with a $P$ value of $<0.05$ in the univariate analysis on mortality were included in a multivariate logistic regression analysis. All statistical analyses were performed with SPSS software (version 21.0; IBM SPSS, Chicago, IL, USA). A $P$ value $<0.05$ was considered significant.

\section{Results}

The clinical and procedural characteristics of the patients are shown in Table 1. Of the 335 patients with acute anterior circu- lation stroke, 233 patients had occlusions in the MCA and 102 in the ICA. Successful revascularization (modified $\mathrm{TICl} 2 \mathrm{~b}$ or 3 ) was achieved in $81.8 \%(n=274 / 335)$ and a good outcome in $45.1 \%$ of patients $(n=151 / 335)$. Parenchymal hemorrhage occurred in $8.9 \%(n=30 / 335)$ and symptomatic hemorrhage in 3.9\% $(n=13 / 335)$. The mortality rate was $10.7 \%(n=36 / 335)$. Seventy-one patients $(21.2 \%)$ received manual aspiration thrombectomy as a rescue therapy after failed stent-retriever thrombectomy. Forty patients (11.9\%) had underlying intracranial atherosclerotic stenosis, and $36(10.7 \%)$ had a tandem occlusion at the proximal cervical portion of the ICA. In the entire cohort $(n=335)$, the median DWI-ASPECTS was 7 (IQR, 6-8). Patients with good outcome had a higher DWI-ASPECTS score compared to those with poor outcome (8 vs. $7 ; O R, 1.278 ; P<0.001$ ).

In univariate analysis, the following variables were identified as predictors of a good outcome at 3 months: younger age, absence of hypertension, DWI-ASPECTS, MCA occlusions (vs. ICA

Table 1. Clinical and procedural characteristics of the study population

\begin{tabular}{|c|c|c|c|c|}
\hline & Total $(n=335)$ & $m R S 0-2(n=151)$ & mRS 3-6 $(n=184)$ & $P$ value \\
\hline Age, year, median (IOR) & $72(64-79)$ & $70(60-76)$ & $74(67-80)$ & $<0.001$ \\
\hline Sex, male & $166(49.6 \%)$ & $82(54.3 \%)$ & $84(45.7 \%)$ & 0.115 \\
\hline \multicolumn{5}{|l|}{ Risk factors } \\
\hline Hypertension & $197(58.8 \%)$ & $79(52.3 \%)$ & $118(64.1 \%)$ & 0.029 \\
\hline Diabetes mellitus & $67(20 \%)$ & $23(15.2 \%)$ & $44(23.9 \%)$ & 0.055 \\
\hline Coronary artery disease & $34(10.1 \%)$ & $17(11.3 \%)$ & $17(9.2 \%)$ & 0.543 \\
\hline Dyslipidemia & $82(24.5 \%)$ & $39(25.8 \%)$ & $43(23.3 \%)$ & 0.603 \\
\hline Smoking & $84(25.1 \%)$ & $41(27.2 \%)$ & $43(23.3 \%)$ & 0.427 \\
\hline Atrial fibrillation & $183(54.6 \%)$ & $75(49.7 \%)$ & $108(58.7 \%)$ & 0.099 \\
\hline Congestive heart failure & $9(2.7 \%)$ & $5(3.3 \%)$ & $4(2.2 \%)$ & 0.736 \\
\hline Previous stroke or TIA & $52(15.5 \%)$ & $18(11.9 \%)$ & $34(18.5 \%)$ & 0.099 \\
\hline Intravenous thrombolysis & $205(61.2 \%)$ & $91(60.3 \%)$ & $114(61.9 \%)$ & 0.810 \\
\hline Occlusion sites & & & & 0.032 \\
\hline ICA & $102(30.4 \%)$ & $37(36.3 \%)$ & $65(63.7 \%)$ & \\
\hline MCA & $233(69.6 \%)$ & $114(48.9 \%)$ & $119(51.1 \%)$ & \\
\hline DWI-ASPECTS & $7(6-8)$ & $8(6-8)$ & $7(5-8)$ & $<0.001$ \\
\hline Time to treatment, minute, median (IOR) & $230(170-310)$ & $220(165-300)$ & $238(180.75-310)$ & 0.115 \\
\hline Procedure time, minute, median (IOR) & $30(20-47)$ & $28(20-40)$ & $35(20.5-50)$ & 0.003 \\
\hline Time to revascularization, minute, median (IOR) & $263(205-340)$ & $250(187-335)$ & $275.5(218.5-347.5)$ & 0.028 \\
\hline Baseline NIHSS score, median (IOR) & $13(10-16)$ & $12(9-15)$ & $14(11-17)$ & $<0.001$ \\
\hline Use of rescue therapy & $71(21.2 \%)$ & $24(15.9 \%)$ & $47(25.5 \%)$ & 0.032 \\
\hline Underlying intracranial stenosis & $40(11.9 \%)$ & $24(15.9 \%)$ & $16(8.7 \%)$ & 0.061 \\
\hline Cervical ICA tandem occlusion & $36(10.7 \%)$ & $19(12.6 \%)$ & $17(9.2 \%)$ & 0.325 \\
\hline \multicolumn{5}{|l|}{ Stroke etiology } \\
\hline Cardioembolism & $198(59.1 \%)$ & $84(55.6 \%)$ & $114(61.9 \%)$ & 0.241 \\
\hline Large artery atherosclerosis & $71(21.2 \%)$ & $41(27.2 \%)$ & $30(16.3 \%)$ & 0.016 \\
\hline Undetermined & $65(19.4 \%)$ & $25(16.6 \%)$ & $40(21.7 \%)$ & 0.233 \\
\hline Others & $1(0.3 \%)$ & $1(0.7 \%)$ & 0 & 0.451 \\
\hline Modified TICl $2 \mathrm{~b}$ or 3 & $274(81.8 \%)$ & $140(92.7 \%)$ & $134(72.8 \%)$ & $<0.001$ \\
\hline Subarachnoid hemorrhage & $66(19.7 \%)$ & $24(15.9 \%)$ & $42(22.8 \%)$ & 0.112 \\
\hline Parenchymal hemorrhage & $30(8.9 \%)$ & $4(2.6 \%)$ & $26(14.1 \%)$ & $<0.001$ \\
\hline Symptomatic hemorrhage & $13(3.9 \%)$ & $1(0.7 \%)$ & $12(6.5 \%)$ & 0.008 \\
\hline
\end{tabular}

mRS, modified Rankin Scale; IQR, interquartile range; TIA, transient ischemic attack; ICA, internal carotid artery; MCA, middle cerebral artery; ASPECTS indicates Alberta Stroke Prognosis Early CT Score; NIHSS, National Institute of Health Stroke Scale; TICl, thrombolysis in cerebral infarction. 
Table 2. Logistic regression analysis of predictors of good outcome

\begin{tabular}{|c|c|c|c|c|c|c|}
\hline & Unadjusted OR & $95 \% \mathrm{Cl}$ & $P$ value & Adjusted OR & $95 \% \mathrm{Cl}$ & $P$ value \\
\hline Age, per 1-year increase & 0.961 & $0.941-0.980$ & $<0.001$ & 0.965 & $0.944-0.986$ & 0.001 \\
\hline Baseline NIHSS, per 1-point increase & 0.891 & $0.843-0.942$ & $<0.001$ & 0.908 & $0.855-0.965$ & 0.002 \\
\hline Hypertension & 0.614 & $0.396-0.952$ & 0.029 & - & - & - \\
\hline MCA vs. ICA occlusion & 1.683 & $1.043-2.716$ & 0.033 & - & - & - \\
\hline DWI-ASPECTS, per 1-point increase & 1.278 & $1.114-1.466$ & $<0.001$ & - & - & - \\
\hline Procedure time, per 1-minute increase & 0.982 & $0.970-0.994$ & 0.003 & - & - & - \\
\hline Time to revascularization, per 1-minute increase & 0.998 & $0.995-1.000$ & 0.046 & - & - & - \\
\hline Use of rescue therapy & 0.551 & $0.319-0.953$ & 0.033 & - & - & - \\
\hline Modified $\mathrm{TICl} 2 \mathrm{~b}$ or 3 & 4.749 & $2.372-9.510$ & $<0.001$ & 4.658 & $2.240-9.689$ & $<0.001$ \\
\hline Large artery atherosclerosis & 1.913 & $1.125-3.253$ & 0.017 & - & - & - \\
\hline Parenchymal hemorrhage & 0.165 & $0.056-0.485$ & 0.001 & 0.150 & $0.049-0.460$ & 0.001 \\
\hline Symptomatic hemorrhage & 0.096 & $0.012-0.744$ & 0.025 & - & - & - \\
\hline
\end{tabular}

$\mathrm{OR}$, odds ratio; $\mathrm{Cl}$, confidence interval; NIHSS, National Institute of Health Stroke Scale; $\mathrm{MCA}$, middle cerebral artery; ICA, internal carotid artery; ASPECTS indicates Alberta Stroke Prognosis Early CT Score; TICl, thrombolysis in cerebral infarction.

Table 3. Logistic regression analysis of predictors of mortality

\begin{tabular}{|c|c|c|c|c|c|c|}
\hline & Unadjusted OR & $95 \% \mathrm{Cl}$ & $P$ value & Adjusted OR & $95 \% \mathrm{Cl}$ & $P$ value \\
\hline Age, per 1-year increase & 1.052 & $1.013-1.091$ & 0.008 & 1.043 & $1.002-1.086$ & 0.041 \\
\hline Baseline NIHSS, per 1-point increase & 1.135 & $1.032-1.247$ & 0.009 & - & - & - \\
\hline Previous stroke/TIA & 3.237 & $1.501-6.984$ & 0.003 & 3.124 & $1.340-7.281$ & 0.008 \\
\hline Modified $\mathrm{TICl} 2 \mathrm{~b}$ or 3 & 0.168 & $0.081-0.348$ & $<0.001$ & 0.171 & $0.079-0.370$ & $<0.001$ \\
\hline Parenchymal hemorrhage & 2.897 & $1.145-7.331$ & 0.025 & 2.961 & $1.059-8.276$ & 0.038 \\
\hline Symptomatic hemorrhage & 5.867 & $1.808-19.038$ & 0.003 & - & - & - \\
\hline
\end{tabular}

OR, odds ratio; $\mathrm{Cl}$ indicates confidence interval; NIHSS, National Institute of Health Stroke Scale; TIA, transient ischemic attack; TICI, thrombolysis in cerebral infarction.

occlusions), low baseline NIHSS, no need for rescue therapy, successful revascularization, absence of parenchymal hemorrhage or symptomatic hemorrhage, shorter procedure time, and a shorter time to revascularization. When dichotomized, patients aged $\geq 80$ years had more frequent poor outcome (mRS 3-6) than those aged $<80$ years $(69.6 \%$ vs. $51.1 \% ; O R, 2.2 ; P=0.007)$ in univariate analysis. In multivariate analysis, younger age $(O R$, $0.965 ; 95 \% \mathrm{Cl}, 0.944-0.986 ; P=0.001$ ), successful revascularization (OR, 4.658; 95\% Cl, 2.240-9.689; $P<0.001$ ), low baseline NIHSS (OR, 0.908; 95\% Cl, 0.855-0.965; $P=0.002)$, and absence of parenchymal hemorrhage $(\mathrm{OR}, 0.150 ; 95 \% \mathrm{Cl}, 0.049-0.460$; $P=0.001)$ were independent predictors of good outcome at 3 months (Table 2).

In univariate analysis, age, history of previous stroke/transient ischemic attack (TIA), revascularization status, symptomatic hemorrhage, and baseline NIHSS score were associated with mortality at 3 months. Patients aged $\geq 80$ years had a higher mortality rate than those aged $<80$ years ( $18.8 \%$ vs. $8.6 \% ; O R, 2.2 ; P=0.015)$ in univariate analysis. In multivariate analysis, age (OR, 1.043; 95\% $\mathrm{Cl}, 1.002-1.086 ; P=0.041$ ), history of previous stroke/TIA (OR, 3.124; 95\% Cl, 1.340-7.281; $P=0.008$ ), revascularization (OR, 0.171; 95\% Cl, 0.079-0.370; $P<0.001)$, and parenchymal hemorrhage $(\mathrm{OR}, 2.961 ; 95 \% \mathrm{Cl}, 1.059-8.276 ; P=0.038)$ were independent predictors of mortality at 3 months (Table 3).

\section{Discussion}

The main findings of our study are summarized as follows: 1) age, revascularization status, and parenchymal hemorrhage are significant independent predictors of not only good clinical outcome but also mortality at 3 months; 2 ) in addition, NIHSS score on admission is independently associated with good outcome, whereas a history of previous stroke/TIA is independently associated with mortality. Several studies have evaluated independent predictors of clinical outcome after stent-retriever thrombectomy in patients with acute ischemic stroke due to intracranial large vessel occlusion. ${ }^{4-8}$ These previous studies have identified that younger age, lower admission NIHSS score, successful recanalization, shorter procedure time, smaller early ischemic changes on

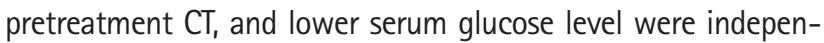
dent predictors of good outcome. ${ }^{4-8}$ Symptomatic ICH, baseline DWI-ASPECT score, baseline NIHSS $\geq 20$, and onset to recanalization $>5$ hours have been identified as independent predictors of mortality after stent-retriever thrombectomy. ${ }^{5.8}$ However, these studies are limited by small sample sizes and inclusion of posterior circulation stroke. The strengths of our study include a large 
sample size, inclusion of only anterior circulation stroke, and consistency in patient selection and endovascular procedures.

In our study, age was significantly associated with clinical outcomes. A good outcome was significantly less frequent among patients $\geq 80$ years than among those $<80$ years $(30.4 \%$ vs. $48.9 \%)$ and mortality was significantly more frequent among patients $\geq 80$ years ( $18.8 \%$ vs. $8.6 \%)$ in the present study. Patient age $\geq 80$ years was associated with a 2.2 -fold increase in both poor outcome and mortality compared with the younger cohort. The results of our study are in agreement with those of North American Solitaire Stent-Retriever Acute Stroke (NASA) registry, which included 354 patients. In the NASA registry, patients $>80$ years showed less favorable outcome $(27.3 \%$ vs. $45.4 \%, P=0.02)$ and increased mortality ( $43.9 \%$ vs. $27.3 \%$, $P=0.01$ ) compared with patients $\leq 80$ years in age. ${ }^{14}$ However, a meta-analysis of 4 recent randomized trials showed a trend toward better outcome (mRS $0-2$ at 90 days; $38 \%$ vs. $19 \%$ ) and a significant reduction in mortality (20\% vs. $41 \%$, adjusted OR 3.7 ; $P=0.01$ ) in patients $\geq 80$ years who received Solitaire stent thrombectomy when compared to those received medical treatment alone. ${ }^{15}$ Thus, old age should not be used as exclusion criteria for stent-retriever thrombectomy in patients with acute large vessel occlusions. . $^{15,16}$

Our study suggests that successful recanalization is still a strong predictor of clinical outcome after endovascular treatment for acute ischemic stroke in this recent era of mechanical thrombectomy. In the present study, successful recanalization (defined as modified $\mathrm{TICl} \geq 2 \mathrm{~b}$ ) occurred in $82 \%$ of patients and was the most powerful independent predictor of both good clinical outcome and mortality. Our results are consistent with 2 previous reports, which demonstrated that successful recanalization is one of the independent predictors of good outcome., With regard to association between recanalization and mortality, the NASA registry showed that successfully recanalized patients had lower mortality (25.2\% vs. $46.9 \%, P<0.001$ in a univariate analysis) after Solitaire stent thrombectomy. ${ }^{16}$ In the NASA registry, proximal occlusion (ICA or basilar artery occlusion), high admission NIHSS score ( $\geq 18$ ), and need for rescue therapy were predictors of mortality in successfully recanalized patients. ${ }^{17}$ The results of our study together with previous studies strongly suggest that neurointerventionalists should make every effort to achieve successful recanalization in order to increase good outcome and decrease mortality.

Parenchymal hemorrhage is considered the most catastrophic complication of reperfusion therapy for acute ischemic stroke and is known to be one of predictors of clinical outcome. ${ }^{18,19}$ In our study, parenchymal hemorrhage occurred in $8.9 \%$ of patients. Patients with parenchymal hemorrhage had good out- come less frequently (13.3\% vs. 48.2\%) and mortality more frequently (23.3\% vs. $9.5 \%)$ compared to those without it. Our findings are consistent with a recent multicenter retrospective study, which included 1,122 patients with anterior circulation large vessel occlusion strokes who received multimodal endovascular therapy. ${ }^{19}$ In that study, parenchymal hemorrhage occurred in $8.6 \%$ of patients (96 of 1122) and was associated with a higher rate of poor outcome $(\mathrm{OR}=6.24, P<0.001)$ and higher mortality $(\mathrm{OR}=3.52, P<0.001)$. In that study, atrial fibrillation $(\mathrm{OR}=1.61, P=0.045)$ was an independent predictor of parenchymal hemorrhage. In addition, Mishra et al. ${ }^{20}$ suggested that the presence of a severely hypoperfused lesion on pretreatment imaging (defined as a very low cerebral blood volume on perfusion MRI) is a strong predictor of parenchymal hemorrhage $(\mathrm{OR}=33, P<0.001)$ in patients undergoing endovascular therapy for acute stroke. However, there was no significant clinical or procedural predictor of parenchymal hemorrhage in univariate or multivariate analysis in our study. Although the occurrence of parenchymal hemorrhage after endovascular therapy is multifactorial, careful patient selection based on pretreatment imaging studies, judicious management of blood pressure, and reduced use of contrast agents and thrombolytic drugs during the endovascular procedure are all needed to decrease the occurrence of parenchymal hemorrhage.

Interestingly, a history of previous stroke/TIA was independently associated with mortality in our study, which has not been previously reported in stent retriever thrombectomy studies. Mortality occurred more frequently in patients with previous stroke/TIA (23.1\% vs. 8.5\%) than those without it. Although this is a novel finding in studies regarding mechanical thrombectomy using stent retrievers, a history of previous stroke/TIA has been found as a predictor of short-term and long-term mortality in patients with acute ischemic stroke compared with healthy control subjects in observational studies. ${ }^{21,22}$ The results of our study and previous studies indicate that more improved management of vascular risks is needed to prevent secondary stroke and subsequent cardiovascular mortality in patients with first-ever stroke.

In our study, NIHSS score on admission was independently associated with good outcome but not with mortality. This finding is consistent with other studies regarding stent-retriever thrombectomy. Costalat et al. ${ }^{4}$ reported that baseline NIHSS score $(\mathrm{OR}=1.228, P=0.002)$ was an independent predictor of poor outcome at 3 months. Jiang et al. ${ }^{8}$ showed that patients with lower baseline NIHSS score (score $<20$ ) had good outcome less frequently $(21.1 \%$ vs. $56.9 \%, O R=5.25)$ compared with those NIHSS score $\geq 20$. In addition, Shi et al. ${ }^{16}$ reported that baseline NIHSS score was an independent predictor of functional depen- 
dence (mRS 3-6) despite successful revascularization $(O R=1.13$, $P=0.0074)$ in 109 patients treated with the Trevo stent-retriever. However, like advanced age, severe stroke should not be used as exclusion criteria because patients with severe stroke do even worse with medical therapy alone. ${ }^{16,23}$

Baseline ASPECTS measured by CT or MRI has been found to be an independent predictor of clinical outcome after stent retriever thrombectomy in two previous studies. ${ }^{5.7}$ In the present study, DWI-ASPECTS was significantly higher in patients with good outcome than those with poor outcome in a univariate analysis $(\mathrm{OR}=1.278$, per 1 -point increase, $P<0.001)$. However, DWI-ASPECTS was not an independent predictor of both good outcome and mortality in multivariate analyses. Similarly, a recent study showed that there were no differences in outcomes between patients with high DWI-ASPECT scores of 7-10 and those with intermediate scores of 4-6 who underwent stent retriever thrombectomy for acute anterior circulation stroke. ${ }^{24}$ This finding may be because of the high rate of successful revascularization with stent retrievers in that study and the disparity between ASPECT score and infarct lesion volume.

Our study has several limitations, including its retrospective and noncontrolled study design. In addition, some potential confounding factors, such as blood glucose level were not analyzed in our study. Admission blood glucose level is known to be associated with poor outcome, increased hemorrhage, and mortality following IV thrombolysis or stent-retriever thrombectomy. ${ }^{4,7,8}$ We did not analyze blood glucose level in this study because it was missing for some patients. Finally, the fact that $21.2 \%$ of patients received manual aspiration thrombectomy as a rescue therapy after failed stent-retriever thrombectomy may limit the accuracy of data in regard to interpretation related to the value of stent retriever thrombectomy.

\section{Conclusions}

In conclusion, age, revascularization status, and parenchymal hemorrhage are independently associated with both good outcome and mortality after stent-retriever thrombectomy in patients with acute anterior circulation stroke. In addition, NIHSS score on admission is independently associated with good outcome, whereas a history of previous stroke is independently associated with mortality. Our results suggest that achieving successful revascularization and trying to reduce parenchymal hemorrhage are priorities when performing stent retriever thrombectomy in patients with acute anterior circulation stroke. The data also suggest a novel finding that a history of previous stroke/TIA can be considered a predictor of mortality in patients receiving stent retriever thrombectomy.

\section{References}

1. Powers WJ, Derdeyn CP, Biller J, Coffey CS, Hoh BL, Jauch EC, et al. 2015 American Heart Association/American Stroke Association Focused Update of the 2013 Guidelines for the Early Management of Patients With Acute Ischemic Stroke Regarding Endovascular Treatment: A Guideline for Healthcare Professionals From the American Heart Association/American Stroke Association. Stroke 2015;46(10):3020-3035.

2. Badhiwala JH, Nassiri F, Alhazzani W, Selim MH, Farrokhyar $F_{\text {, }}$ Spears J, et al. Endovascular Thrombectomy for Acute Ischemic Stroke: A Meta-analysis. JAMA. 2015;314(17):1832-1843.

3. Bush CK, Kurimella D, Cross $\sqcup$, Conner KR, Martin-Schild S, He $J$, et al. Endovascular Treatment with Stent-Retriever Devices for Acute Ischemic Stroke: A Meta-Analysis of Randomized Controlled Trials. PLoS One 2016;11(1):e0147287.

4. Costalat V, Lobotesis K, Machi P, Mourand I, Maldonado I, Her-

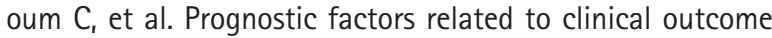
following thrombectomy in ischemic stroke (RECOST study). 50 patients prospective study. Eur J Radiol 2012;81(12):40754082.

5. Soize S, Barbe C, Kadziolka K, Estrade L, Serre I, Pierot L. Predictive factors of outcome and hemorrhage after acute ischemic stroke treated by mechanical thrombectomy with a stent-retriever. Neuroradiology 2013;55(8):977-987.

6. Daou B, Chalouhi N, Starke RM, Dalyai R, Hentschel K, Jabbour $P$, Rosenwasser R, Tjoumakaris SI. Predictors of Outcome, Complications, and Recanalization of the Solitaire Device: A Study of 89 Cases. Neurosurgery 2015;77(3):355-360.

7. Ozdemir O, Giray S, Arlier Z, Baş DF, Inanc Y, Colak E. Predictors of a Good Outcome after Endovascular Stroke Treatment with Stent Retrievers. Scientific World Journal 2015;2015:Article ID 403726.

8. Jiang S, Fei A, Peng Y, Zhang J, Lu YR, Wang HR, et al. Predictors of Outcome and Hemorrhage in Patients Undergoing Endovascular Therapy with Solitaire Stent for Acute Ischemic Stroke. PLoS One 2015;10(12):e0144452.

9. Clark WM, Wissman S, Albers GW, Jhamandas JH, Madden KP, Hamilton S. Recombinant tissue-type plasminogen activator (alteplase) for ischemic stroke 3 to 5 hours after symptom onset: the ATLANTIS Study: a randomized controlled trial. JAMA. 1999;282(21):2019-2026.

10. Yoon W, Jung MY, Jung SH, Park MS, Kim JT, Kang HK. Subarachnoid hemorrhage in a multimodal approach heavily weighted toward mechanical thrombectomy with Solitaire stent in acute stroke. Stroke 2013;44(2):414-419.

11. Kim SK, Yoon W, Moon SM, Park MS, Jeong GW, Kang HK. Outcomes of manual aspiration thrombectomy for acute ischemic 
stroke refractory to stent-based thrombectomy. J Neurointerv Surg. 2015;7:473-477.

12. Zaidat 00, Yoo AJ, Khatri P, Tomsick TA, von Kummer R, Saver $J L$, et al. Recommendations on angiographic revascularization grading standards for acute ischemic stroke: a consensus statement. Stroke 2013;44(9):2650-2663.

13. Fiorelli M1, Bastianello S, von Kummer R, del Zoppo GJ, Larrue $V$, Lesaffre $E_{\text {, et }}$ al. Hemorrhagic transformation within 36 hours of a cerebral infarct: relationships with early clinical deterioration and 3-month outcome in the European Cooperative Acute Stroke Study I (ECASS I) cohort. Stroke 1999;30(11): 2280-2284.

14. Castonguay AC, Zaidat 00, Novakovic R, Nguyen TN, Taqi MA, Gupta $R$, et al. Influence of age on clinical and revascularization outcomes in the North American Solitaire Stent-Retriever Acute Stroke Registry. Stroke 2014;45(12):3631-3636.

15. Campbell BC, Hill MD, Rubiera M, Menon BK, Demchuk $A$, Donnan GA, et al. Safety and Efficacy of Solitaire Stent Thrombectomy: Individual Patient Data Meta-Analysis of Randomized Trials. Stroke 2016;47(3):798-806.

16. Shi ZS, Liebeskind DS, Xiang B, Ge SG, Feng L, Albers GW, et al. Predictors of functional dependence despite successful revascularization in large-vessel occlusion strokes. Stroke 2014;45 (7):1977-1984.

17. Linfante I, Walker GR, Castonguay AC, Dabus G, Starosciak $A K_{\text {, }}$ Yoo AJ, et al. Predictors of Mortality in Acute Ischemic Stroke Intervention: Analysis of the North American Solitaire Acute Stroke Registry. Stroke 2015;46(8):2305-2308.
18. Mokin M, Kan P, Kass-Hout T, Abla AA, Dumont TM, Snyder KV, et al. Intracerebral hemorrhage secondary to intravenous and endovascular intraarterial revascularization therapies in acute ischemic stroke: an update on risk factors, predictors, and management. Neurosurgical Focus 2012;32(4):E2.

19. Nogueira RG, Gupta R, Jovin TG, Levy El, Liebeskind DS, Zaidat 00 , et al. Predictors and clinical relevance of hemorrhagic transformation after endovascular therapy for anterior circulation large vessel occlusion strokes: a multicenter retrospective analysis of 1122 patients. J Neurointerv Surg. 2015;7(1):16-21.

20. Mishra NK, Christensen S, Wouters A, Campbell BC, Straka M, Mlynash $\mathrm{M}$, et al. Reperfusion of very low cerebral blood volume lesion predicts parenchymal hematoma after endovascular therapy. Stroke 2015;46(5):1245-1249.

21. Hankey GJ. Long-term outcome after ischaemic stroke/transient ischaemic attack. Cerebrovasc Dis 2003;16(Suppl 1):1419.

22. Carter AM, Catto AJ, Mansfield MW, Bamford JM, Grant PJ. Predictive variables for mortality after acute ischemic stroke. Stroke 2007;38(6):1873-1880.

23. Broderick JP, Palesch YY, Demchuk AM, Yeatts SD, Khatri $P$, Hill $M D$, et al. Endovascular therapy after intravenous t-PA versus t-PA alone for stroke. N Engl J Med 2013;368(10):893-903.

24. Kim SK, Yoon W, Park MS, Heo TW, Baek BH, Lee YY. Outcomes are not different between patients with intermediate and high DWI-ASPECTS after stent-retriever embolectomy for acute anterior circulation stroke. AJNR Am J Neuroradiol 2016;37: 1080-1085. 